\title{
Strategies for Effective Management of Intellectually Disabled Patients on the Psychiatric Inpatient Unit
}

\author{
Luisa Gonzalez, Ifeoma Nwugbana, Rahulkumar Patel, Marissa Lombardo and \\ Panagiota Korenis
}

\author{
Department of Psychiatry and Behavioral Sciences Albert, Bronx Lebanon Hospital Center, Einstein College \\ of Medicine, USA
}

\begin{abstract}
The management of aggressive behavior remains a fundamental challenge when working on a psychiatric inpatient service. The task becomes far more daunting when the patient presents not only with mental illness but also has an intellectual disability (ID) or impulse control disorder (IC). Intellectual Disability is defined as "the impairment of general mental abilities that impact adaptive functioning in three domains: conceptual, social and practical." Impulse control disorder, is defined as "a psychiatric disorder characterized by impulsivity- the failure to resist a temptation, urge or impulse that may harm oneself or others" [1]. Those with ID and or IC may present with varying degrees of impairment and social functioning. Numerous studies have identified an association with ID and psychiatric co-morbidities including: bipolar disorder, impulse control disorder, psychosis and depression. Due to budgetary cuts and the precipitous decline in available residential placements, inpatient psychiatric services are faced with the dilemma of managing these exceptionally complicated patients. While numerous studies have examined the utility of psychotropic medication to aid in the management of these patients, convincing evidence concerning the use of psychiatric medication in the management of this patient population remains elusive [2]. Therefore, this paper aims to explore the treatment strategies available to the multidisciplinary team on the inpatient service. Ultimately, future investigations will be necessary to better understand how to optimize the inpatient management of this complex patient population.
\end{abstract}

Keywords: Intellectual Disability, Impulse control disorder, Agitation, Inpatient psychiatry.

\section{INTRODUCTION}

Intellectual disability(ID) (intellectual developmental disorder) is a disorder with onset during the developmental period that includes both intellectual and adaptive functioning deficits in conceptual, social, and practical domains. The three criteria of deficits in intellectual functions, deficits in adaptive functioning and onset of intellectual and adaptive deficits during the developmental period need to be met for this diagnosis to be made. The prevalence of intellectual disability in the general population is estimated at approximately $1 \%$ [3]. The prevalence of mental illness in children and adults with ID is greater than that found in the general population [4]. Cooper et al. found a point prevalence of mental illness in a sample of intellectually disabled individuals of $15.7 \%$ by DSM IVTR criteria [5]. Affective disorders like depression, mania and bipolar depression have been described in this population with point prevalences of $3.8 \%, 0.6 \%$ and $1.0 \%$ respectively [6]. Anxiety disorders have been found to be just as common, a study finding a prevalence rate of $3.8 \%$ [7]. A higher prevalence and incidence of psychosis in patients with intellectual disability compared to the general population is well documented [8]. Some studies have also shown that

*Address correspondence to this author at the Department of Psychiatry and Behavioral Sciences Albert, Bronx Lebanon Hospital Center, Einstein College of Medicine, USA; Tel: 718-901-6265/914-426-5127; Fax: 718-901-8656;

E-mail: pkorenis@bronxleb.org those who present with both ID and impulse control disorder or bipolar disorder have a tendency to be more aggressive, with a point prevalence of $9.8 \%$ found in a cohort of patients using the Diagnostic Criteria for Psychiatric Disorders for Use with Adults with Learning Disabilities/Mental Retardation (DC-LD) diagnosis [9]. Impulse control disorders and aggressiveness are the main reasons inpatient psychiatric hospitalization is sought for people with intellectual disability. Comprehensive management of this unique population in a psychiatric inpatient setting is underreported in the literature and so mental health personnel usually feel ill equipped to deal with the challenges posed by managing them. There is also a significant drain on public resources as their hospitalizations are usually associated with numerous barriers to dispositon leading to increased length of stay. Specialized training of mental health personnel is needed in order to effectively manage these patients.

\section{DISCUSSION}

Patients with ID and IC on Psychiatric Inpatient Units are managed in a multidisciplinary fashion. Members of this multidisciplinary approach include psychiatrists, social work, nursing staff, psychologists (if consulted on the case), the patient and the patient's family/care giver. Additionally, incorporating a medical doctor is also particularly important in managing these patients as they are at high risk for various infections, trauma secondary to self-injurious behavior and other 
medical complications secondary to globally limited insight into their behavior. Best practices for management of such complex patients include mental health professionals as part of a team that includes behavioral psychologists, occupational therapists, physical therapists, pediatric, neurology, medicine, psychiatry, educators, family members, and direct support professionals. The goal is to effectively integrate different points of view and treatment models into a meaningful treatment plan for the patient.

Studies suggest that the most common symptoms on admission include psychotic disorganization, withdrawal and bizarre rituals [10]. Although only one small example of a much larger issue that needs to be addressed, it is clear that a multidisciplinary approach is important and effective in managing a patient with ID and psychiatric disturbance. Since there are many issues outside of the primary psychiatric diagnosis in these patients such as the consideration of his/her activity of daily living, functioning, socioeconomic status and the strong need for family/caregiver support, these patients should have a specialized and highly adapted management approach to the inpatient care.

The psychiatric inpatient unit is an environment in which staff is trained to deal with patients with mental illness. Now, more frequently, staff is being faced with addressing the needs of patients with co-occurring mental illness and developmental disability who present with behavioral problems and psychiatric symptoms unmanageable in the community. While it is challenging to work daily with a psychiatric patient, it is even more challenging to manage a person with developmental disability and co-occurring psychiatric presentation without having proper training.

Having a patient admitted to a specialized intellectual disability unit which has a specialized intellectual psychiatric team composed of psychiatrists, nurses, support staff, behavioral psychologists, social workers, a recreational therapist, an occupational and a speech therapist is ideal but clearly not the norm. More frequently, these individuals are admitted on a general inpatient psychiatric unit that does not have special facilities or trained staff specialized in intellectual disability. This can lead to significantly longer lengths of stay in the hospital.

\section{WHAT CAN BE DONE ON THE UNIT?}

On initial presentation, the treatment team must work together with family members or staff at the residence where the person is residing in order to obtain reliable and accurate information. It is important to obtain the patient's baseline behavior, behavioral treatment modalities used in the past, crisis plans, documentation of known antecedent events and effective de-escalation methods. A picture of the individual's daily routine activity (i.e. eating and sleeping patterns) can help the psychiatrist and nursing staff have a better understanding in determining whether the patient is now presenting with something totally new, the exacerbation of an existing behavior or a manifestation of an underlying medical condition. Clinician should familiarize themselves with common co-occurring medical conditions because many people with developmental disability have limited verbal skills. Pain and discomfort may make them physically aggressive and delay appropriate medical care, leading to misdiagnosis, unnecessary medication administration and further prolong their length of stay.

All staff should focus on establishing rapport and communicating properly with these individual by avoiding medical jargon, and allowing additional time for the patient to respond to questions asked or to cooperate with assessments. The team can identify perhaps one or two support staff member that work on different shifts to be always present, that work closely and have built trust with these individuals with the expectation that the patient will cooperate further with there treatment.

Documentation by all staff daily, not only clinical staff but also the support staff/patient care technician who are observing, and assisting directly with the patient during their activity of daily living is vital. Documentation of the patient's ability to take care of personal hygiene, grooming and eating habits, as well as cooperation with routine procedures, can provide information to the clinical team regarding the patient's capabilities. This avoids assuming or having higher expectations from the patient, and prompts the patient to maintain their current level of functioning, thus avoiding regression. Daily documentation can also help in making appropriate and tailored treatment plan objectives for the patient.

\section{ENCOURAGE ACTIVE PARTICIPATION}

It is important that the patient continue to follow a structured routine, ideally similar to what they have been receiving in their home/residence, day program in the community. This of course should be tailored to the new environment in an attempt to be minimally disruptive. Specialized health care professionals should encourage patient with ID to actively participate 
in group activity such as Anger Management, Women's/Men's Groups, Coping Skills Groups and Family Support Group, as well as other groups provided on the unit. These groups should be modified for the patient with limited communication skills through art, music or play therapy.

\section{TIPS FOR STAFF}

One of the first things the staff should be informed of is the potential challenges of working with a person with developmental disability. The staff must also have a frame of reference on behaviors and level of functioning that this person may exhibit. Staff would benefit from education related to the functionality of those with developmental disability in order to ensure that they understand what level the patient will be able to function. Such training would ensure that staff has reasonable expectations in terms of activities of daily living and functioning for patients with ID.

All staff should be trained to identify past trauma, understand its effects on people with co-occurring developmental disabilities and mental illness, ensure appropriate adjustments to treatment/service plans to address trauma issues, and avoid re-traumatization [11]. All staff should be informed about the patient's medication regimen, specifically when changes are being made to help identify and track specific behaviors with the objective of evaluating medication efficacy, as well as monitoring for side effects.

Consultation with cross-trained staff (i.e. psychiatrist, developmental disability nurse, behavioral analyst) who are skilled and experienced in working with people with both developmental and mental health issues, can help the treatment team develop an appropriate plan for the patient. They can provide strategies on medication management, crisis management and prevention, as well as teaching staff how to break complex tasks down into small, discrete, and concrete steps that comprise clear instructions for the patient.

When faced with the treatment of a challenging and difficult patient, staff should be cognizant of the counter transference feelings that may result. Staff should be encouraged to verbalize these feeling to the supervisor that can in turn provide counseling to help staff maintain composure, control anger, and develop coping skills to manage challenging situations.

Supervisors must also be mindful of employee burnout and should educate staff about coping skills.
Staff should receive feedback and guidance on how to deal calmly and effectively in high stress situations. They should be informed that this is going to be a collective team effort, and that every team member is expected to be reliable, responsible and dependable in fulfilling their obligations. At the same time, it is imperative to emphasize the role of the team and that no staff member should bear the burden of managing such challenging patients on their own.

Treatment team leaders should periodically hold meetings with staff to discuss challenges and provide resources for team members or alternative methods of handling problems. They should welcome suggestions and input from all members who are working on the case to provide better care and treatment to the patient. Finally, ongoing staff education and recognition for their efforts and hard work should be provided during and after the patient has been discharged.

\section{CONCLUSION}

Patients with intellectual disability and coexisting psychiatric illness pose a unique challenge to the mental health professional. The literature encourages managing these patients in a multidisciplinary manner with ongoing training and monitoring of staff burnout. The incorporation of many different patient care team players (psychiatrists, psychologists, financial/welfare and family members) increases patient care outcomes and leads to a more effective approach in treating these patients. In general, management of these patients is most successful when various different specialties and subspecialties are involved to create an effective treatment model for patients that require such an extensively complex treatment plan. These patients have an extensively complex set of conditions that go beyond the dual diagnosis and permeate all aspects of their lives. Managing all of these aspects is difficult since there is little understood about the management of these patients at this time. There is essentially very little data to support or to refute any of the current models in place at most psychiatric inpatient facilities.

A multidisciplinary, functionally communicative treatment team with well-defined goals and familial/care giver participation actively in place seems to be the most effective means for treating these patients and improving patient outcomes.

\section{REFERENCES}

[1] American Psychiatric Association. Diagnostic and Statistical Manual of Mental disorders. $5^{\text {th }}$ Edition. Washington, DC: APA Press 2013 
[2] Tyrer P, Oliver-Africano PC, Ahmed Z, Bouras N, Cooray S, Deb S, Murphy D, Hare M, Meade M, Reece B, Kramo K, Bhaumik S, Harley D, Regan A, Thomas D, Rao B, North B, Eliahoo J, Karatela S, Soni A, Crawford M. Risperidone, haloperidol and placebo in the treatment of aggressive challenging behavior in patients with intellectual disability: a randomized control trial. Lancet 2008; 371(9606): 57-63. http://dx.doi.org/10.1016/S0140-6736(08)60072-0

[3] Maulik PK, Mascarenhas M, Mathers C, Dua T, Saxena S. Prevalence of intellectual disability: A meta-analysis of population-based studies. Res Dev Disabil 2011; 32: 419-36. http://dx.doi.org/10.1016/j.ridd.2010.12.018

[4] Mohr C, Tonge BJ, Einfeld SL. The development of a new measure for the assessment of psychopathology in adults with intellectual disability. J Intellect Disabil Res 2005; 49: 469-80. http://dx.doi.org/10.1111/j.1365-2788.2005.00701.x

[5] Cooper SA, Smiley E, Morrison J, Williamson A, Allan L. Mental ill-health in adults with intellectual disabilities: prevalence and associated factors. $\mathrm{Br} \mathrm{J}$ Psychiatry 2007; 190: $27-35$ http://dx.doi.org/10.1192/bjp.bp.106.022483

[6] Cooper SA. An epidemiological investigation of affective disorders with a population-based cohort of 1023 adults with intellectual disabilities. Psychol Med 2007; 37: 873-82. http://dx.doi.org/10.1017/S0033291707009968
[7] Reid KA, Smiley E, Cooper SA. Prevalence and associations of anxiety disorders in adults with intellectual disabilities. J Intellect Disabil Res 2011; 55: 172-81. http://dx.doi.org/10.1111/j.1365-2788.2010.01360.x

[8] Cooper SA, Smiley E, Jackson A, Finlayson J, Allan L, Mantry D and Morrison J. Adults with intellectual disabilities: prevalence, incidence and remission of aggressive behaviour and related factors. J Intellect Disabil Res 2009; 53: 217-32. http://dx.doi.org/10.1111/j.1365-2788.2008.01127.x

[9] Cooper SA, Elita S, Jillian M, Linda A, Andrew W, Janet F, Alison J, Dipali M. Psychosis and adults with intellectual disabilities. Prevalence, incidence, and related factors. Soc Psych Psych Epid 2007; 42: 530-6 http://dx.doi.org/10.1007/s00127-007-0197-9

[10] Myrbakk E, von Tetzchner S: The Prevalence of Behavioral Problems among People with Intellectual Disability Living in Community Settings. J Mental Health Research in Intellectual Disabilities 2008; 1: 205-222. http://dx.doi.org/10.1080/19315860802115607

[11] Serving Individuals with Co-occurring developmental disabilities and mental illnesses: Systems barriers and strategies for reform. National Association of State Mental Health Program Directors (NASMHPD) October 2004.

\section{DOI: http://dx.doi.org/10.6000/2292-2598.2015.03.02.7}

(c) 2015 Gonzalez et al.; Licensee Lifescience Global.

This is an open access article licensed under the terms of the Creative Commons Attribution Non-Commercial License (http://creativecommons.org/licenses/by-nc/3.0/) which permits unrestricted, non-commercial use, distribution and reproduction in any medium, provided the work is properly cited. 